\title{
Usefulness and Cost-effectiveness of Interval Appendectomy for Complicated Appendicitis
}

\author{
Ryosuke Kita $^{1,}$, , Hiroki Hashida ${ }^{1}$, Daisuke Yamashita ${ }^{2}$, Hiromitsu Kinoshita ${ }^{3}$, Masato Kondo ${ }^{1}$, \\ Satoshi Kaihara ${ }^{1}$ \\ ${ }^{1}$ Department of Surgery, Kobe City Medical Center General Hospital, Kobe, Japan \\ ${ }^{2}$ Department of Pathology, Kobe City Medical Center General Hospital, Kobe, Japan \\ ${ }^{3}$ Department of Gastroenterological Surgery, Kyoto University Hospital, Kyoto, Japan
}

Email address:

ryosuke_kita@kcho.jp (R. Kita)

*Corresponding author

\section{To cite this article:}

Ryosuke Kita, Hiroki Hashida, Daisuke Yamashita, Hiromitsu Kinoshita, Masato Kondo, Satoshi Kaihara. Usefulness and Cost-effectiveness of Interval Appendectomy for Complicated Appendicitis. Journal of Surgery. Vol. 9, No. 3, 2021, pp. 109-113. doi: 10.11648/j.js.20210903.13

Received: April 7, 2021; Accepted: April 28, 2021; Published: May 14, 2021

\begin{abstract}
Background: The management for abscess-forming appendicitis, including interval appendectomy, is controversial. Emergency surgery for abscess-forming appendicitis may lead to expanded surgery, which is associated with many perioperative complications. To prevent complications, we introduced the Interval Appendectomy protocol (IA protocol), and examined its efficacy compared to emergency appendectomy. Methods: Patients treated for complicated appendicitis at our hospital from January 2010 to January 2018 were consecutively enrolled into this study. They were classified into two groups based on enrollment before and after April 2014: emergency appendectomy group (EA group, before April 2014) and interval appendectomy group (IA group, after April 2014). We compared the perioperative treatment outcomes and examined the necessity for elective surgery, focusing on the postoperative pathological results of the IA group. Results: We enrolled 49 consecutive patients with complicated appendicitis who were treated during this study period. The IA protocol was applied to 38 patients; 32 patients completed this protocol and were assigned to the IA group. Patient characteristics were not significantly different between the IA and EA group. The IA group had significantly less postoperative complications $(\mathrm{p}=0.002)$ and medical costs $(\mathrm{p}=0.01)$. Residual inflammation in the appendix was observed in 16 cases $(50 \%)$ pathologically. Conclusions: Interval appendectomy for complicated appendicitis was associated with a low incidence of perioperative complications and was effective in terms of cost. Interval appendectomy seems necessary to prevent recurrent appendicitis, given that inflammation remains on pathological examination.
\end{abstract}

Keywords: Interval Appendectomy, Complicated Appendicitis, Perioperative Complications, Cost-effectiveness

\section{Introduction}

About 2 to $6 \%$ of appendicitis form a mass or abscess, and these appendicitis are called complicated appendicitis [1-3]. Emergency surgery for abscess-forming appendicitis may lead to expanded surgery, which is associated with many perioperative complications. The reported rates of perioperative complications in patients undergoing emergency appendectomy for complicated appendicitis range from 5 to $10 \%[4,5]$. Previous studies in pediatrics showed that interval appendectomy was effective and had few complications [6-9]. However, there are also reports that surgery is not recommended after conservative treatment because of non-negligible complication rates of interval appendectomy (9-19\%) [10-12]. Further, its effectiveness for complicated appendicitis in adults remains unclear, and the necessity for elective surgery after conservative treatment remains controversial.

To prevent the transition to expanded surgery and postoperative complications, we introduced the Interval Appendectomy protocol (IA protocol) for complicated appendicitis from April 2014. In this study, we investigated the efficacy of interval appendectomy, compared to emergency appendectomy, for abscess-forming appendicitis in 
adults in terms of medical and economic outcomes, and focused on the necessity of elective surgery from a pathological point of view.

\section{Materials \&Methods}

Patients treated for complicated appendicitis at our hospital from January 2010 to January 2018 were consecutively enrolled into this study. Eligibility criteria for our IA protocol included: i) liquid retention or tumor formation localized $1 \mathrm{~cm}$ or more around the appendix as noted by computed tomography (CT) and ii) non-generalized peritonitis (Figure 1); the presence or absence of coprolite and the age of patients were not considered. Patients were excluded if: i) vital signs were unstable, ii) patients had a comorbidity/condition that made surgery a priority such as chronic renal failure or pregnancy; and iii) artificial objects were placed in the body such as intravascular stents, central venous port, or pace maker. The IA protocol consisted of the following steps (Figure 2). In the first admission, patients were treated conservatively with antibiotic administration and percutaneous drainage in possible cases. After discharge we performed colonoscopy to exclude malignancy, and follow-up CT to check for abscess disappearance. Three months later (two months in coprolite cases), appendectomy was performed. The study protocol was approved by the Institutional Review Board of Kobe City Medical Center General Hospital. Written informed consent was obtained from all patients who underwent our IA protocol.

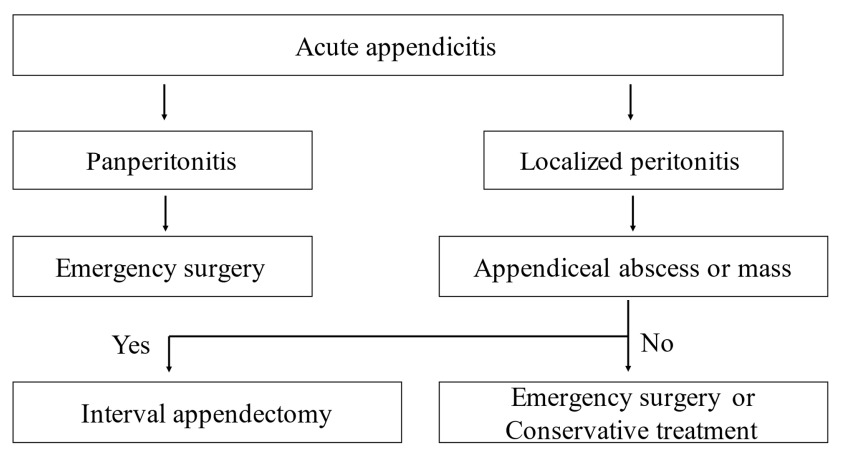

Figure 1. Eligibility criteria

\begin{tabular}{|c|c|}
\hline \multirow[t]{2}{*}{0 months } & $\begin{array}{l}\text { 《First admission 》 } \\
\text { Antibiotics } \pm \text { percutaneous drainage }\end{array}$ \\
\hline & Abdominal condition improves $\Rightarrow$ leave hospital \\
\hline 1 month & Colonoscopy \\
\hline & CT follow-up before readmission \\
\hline 3 months & $\begin{array}{l}\text { 《Second admission》 } \\
\text { Operation "Interval appendectomy" }\end{array}$ \\
\hline
\end{tabular}

CT: computed tomography.

Figure 2. Protocol of interval appendectomy.

Before applying the IA protocol, patients with complicated appendicitis underwent emergency surgery. After April 2014 when the IA protocol started, all patients who had appendicitis with abscess formation were included in this protocol. We classified these patients into two groups as follows: emergency appendectomy group (EA group) and interval appendectomy group (IA group). We compared the perioperative treatment results such as operation time, blood loss, operative method, postoperative complications, hospital stay, and total medical expense between the two groups. Further we examined the necessity for elective surgery focusing on the postoperative pathological results of the IA group.

Continuous variables are presented as median [range], and categorical variables as number and percentage. We conducted a retrospective analysis of the efficacy of our IA protocol. Statistical analysis was performed using Fisher's exact test and Mann-Whitney U-test. All statistical analyses were conducted by one of the physicians participating in the study (RY) using JMP version 12 (SAS Institute Inc., Cary, $\mathrm{NC}$, USA). A p-value of $<0.05$ was considered to indicate a statistically significant difference.

\section{Results}

We enrolled 49 consecutive patients with complicated appendicitis who were treated during this study period. Before starting the IA protocol, 11 patients underwent emergency surgery and were assigned to the EA group. The IA protocol was applied to 38 patients, but 4 dropped out of this protocol and shifted from conservative treatment to emergency surgery because 3 patients showed the exacerbation of abdominal findings and deteriorating inflammatory response in blood examination, and 1 patient experienced recurrent appendicitis as an outpatient after successful conservative treatment. The colonoscopy which was performed before elective surgery revealed cecal and appendiceal cancer in 2 patients, so 32 patients completed this protocol and were assigned to the IA group. The patient selection is shown in Figure 3.

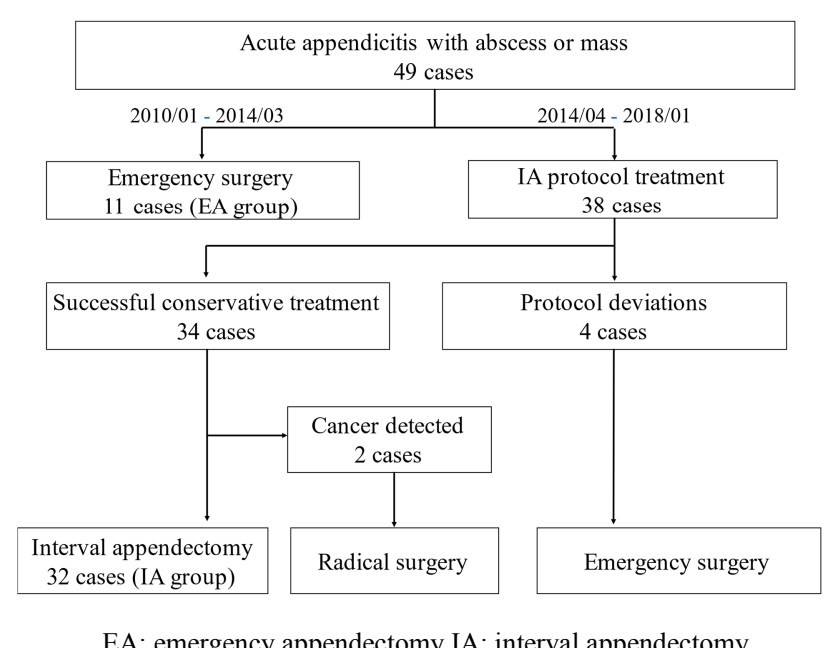

Figure 3. Patient selection.

A comparison of the characteristics of the EA and IA group is presented in Table 1. The IA group tended to have fewer 
cases with coprolite, but there was no significant difference in almost all the characteristics considered. Interval appendectomy was performed at 97 days after discharge (range, 55-319 days).

Table 1. Comparison of the patient characteristics of the two groups.

\begin{tabular}{llll}
\hline Variable & EA $(\mathbf{n}=\mathbf{1 1})$ & IA $(\mathbf{n}=\mathbf{3 2})$ & p-value \\
\hline Sex, Male/Female & $4 / 7$ & $16 / 16$ & 0.499 \\
Age $($ years $)$ & $38[15-61]$ & $53[40-68]$ & 0.068 \\
BMI $\left(\mathrm{kg} / \mathrm{m}^{2}\right)$ & $19.7[17.8-22.1]$ & $21.5[20.3-23.2]$ & 0.096 \\
WBC $(/ \mu \mathrm{L})$ & $16000[12400-17600]$ & $14150[12125-16750]$ & 0.427 \\
CRP $(\mathrm{mg} / \mathrm{dL})$ & $16.4[10.5-20.3]$ & $13.6[10.4-18.9]$ & 0.370 \\
Mass diameter & $40[32-48]$ & $41.5[32-55.3]$ & 0.814 \\
$(\mathrm{~mm})$ & $6 / 5$ & $6 / 26$ & 0.041 \\
Coprolite yes/no & $6 / 5$
\end{tabular}

Values are presented as median [interquartile range] or as numbers.

EA: emergency appendectomy; IA: interval appendectomy; BMI: body mass index; WBC: white blood cell; CRP: C-reactive protein

Table 2. Comparison of the perioperative results between the two groups.

\begin{tabular}{llll}
\hline Variable & EA $(\mathbf{n}=\mathbf{1 1})$ & $\mathbf{I A ~ ( n = 3 2 )}$ & p-value \\
\hline Operation time (min) & $151[114-188]$ & $88[43-107.3]$ & 0.001 \\
Blood loss (mL) & $317[90-380]$ & $0[0-100]$ & $<0.001$ \\
Operative method & & & \\
Laparoscopy & 2 & 32 & \\
Conversion to laparotomy & 3 & 0 & $<0.0001$ \\
Laparotomy & 6 & 0 & \\
Appendectomy & 3 & 32 & $<0.0001$ \\
Cecum partial resection & 2 & 0 & \\
Ileocecal resection & 6 & 0 & \\
Complications & & & 0.002 \\
SSI & 3 & 2 & \\
Abscess formation & 1 & 0 & \\
Ileus & 1 & 0 & \\
Hospital stay (days) & & & \\
First admission & 11 & 10 & \\
Second admission & 0 & 3 & \\
Total & $11[8-15]$ & $13[11-16]$ & \\
Medical expense (\$) & & 4687 & \\
First admission & 10862 & 3749 & \\
Second admission & 0 & 8436 & \\
Total & 10862 & & \\
\hline
\end{tabular}

Values are presented as median [interquartile range] or as numbers

EA: emergency appendectomy; IA: interval appendectomy; SSI: surgical site infection

Comparison of perioperative results between the EA and IA group is shown in Table 2. The total length of stay was similar between both groups (11 days in the EA group vs. 13 days in the IA group, $\mathrm{p}=0.367)$. The majority $(81.8 \%)$ of 11 surgeries in the EA group were laparotomy or laparotomy conversion (9 procedures) and there was a tendency for enlargement surgery such as ileocecal resection. All procedures in the IA group were laparoscopic appendectomy. Therefore, operation time and blood loss were significantly less in the IA group (151 $\mathrm{min} / 317 \mathrm{~mL}$ in the EA group vs. $88 \mathrm{~min} / 0 \mathrm{~mL}$ in the IA group, $\mathrm{p}=0.001 /<0.001$ ). Only 2 patients developed superficial surgical site infection in the IA group; the IA group had significantly less postoperative complications than the EA group $(p=0.002)$. Moreover, total medical expenses were significantly lower in the IA group ( $\$ 10862$ in the EA group vs. $\$ 8436$ in the IA group).
Pathological findings of appendectomy specimens in the IA group showed residual inflammation in the appendix in 16 cases $(50 \%)$. Lymphocytes and plasma cells were found instead of neutrophils, and they were in a state of transition to chronic inflammation (Figure 4). However, in some cases, the accumulation of neutrophils and survival of abscesses were observed (Figure 5). (a)

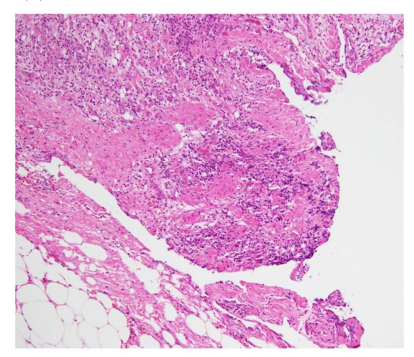

(b)

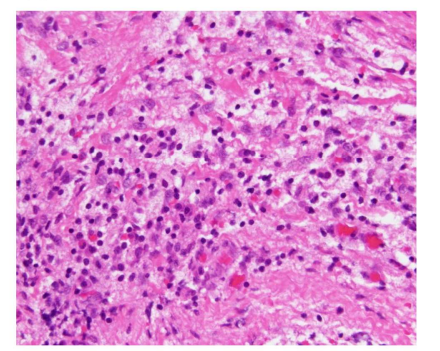

Figure 4. Pathological finding of appendectomy specimens in the IA group (HE).

(a): Fat necrosis and reactive fiberous hyperplasia existed mainly in the subserosa $(\times 10)$.

(b): Lymphocytes and plasma cells were observed $(\times 40)$. (a)

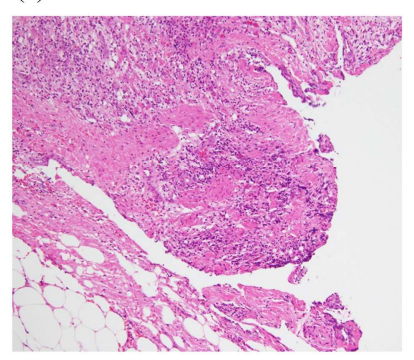

(b)

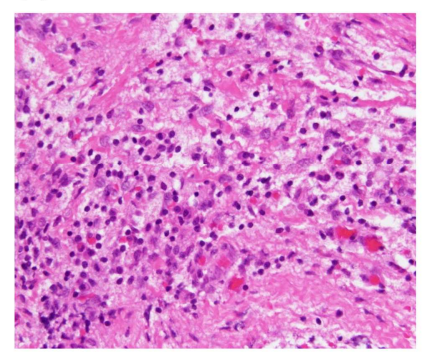

Figure 5. Pathological finding of appendectomy specimens in the IA group (HE).

(a): Survival of abscesses was observed $(\times 10)$.

(b): Accumulation of neutrophils was observed $(\times 40)$.

\section{Discussion}

Although antibiotic therapy as a first treatment is considered more effective than emergency appendectomy for appendicitis with abscess formation, the management of appendicitis with abscess formation, including interval appendectomy, has been controversial [13-16]. Our study showed a low incidence of perioperative complications for interval appendectomy for complicated appendicitis. The low complication rate was due to the appropriate timing of elective surgery; it is considered that 3 months is the time for disappearance of adhesions which make surgery difficult and lead to postoperative complications. Lai et al reported that routine interval appendectomy was not effective in terms of costs [17]. However, our study showed the significantly better cost-effectiveness of interval appendectomy compared to emergency appendectomy. The reason was that in the EA group, the costs for emergency surgery and the management 
of postoperative complications prolonged hospitalizations and increased the total medical expense, while in the IA group, the cost was reduced due to the negligible complications despite two hospitalizations.

A high probability of protocol success (88.9\%) was observed. However, regarding the dropout cases of IA group in our study, all 4 cases showed abscess formation at the site where percutaneous drainage was difficult. Percutaneous drainage was more effective than antibiotics alone in treating patients $[18,19]$. The drainage of abscess can reduce the spread of inflammation around the area and prevent generalized peritonitis. In this study, CT-guided drainage was performed in $33 \%$ of all cases, and aggressive drainage was considered to increase the success rate of this protocol. In addition, cancer was detected in 2 cases, and radical resection was performed. By performing colonoscopy after conservative treatment, cancer can be ruled out and unnecessary additional resection can be avoided. Both patients experienced good progression without recurrence for 1 year after surgery.

Residual inflammation has been reported in resected specimens after interval appendectomy [20, 21]. According to research results in our hospital, remnant pathological inflammation was observed in half of the patients. The transition from acute inflammation to chronic inflammation was observed. In some cases, acute inflammation and abscess remained even after 3 months. The inflammation remained even when blood and imaging tests became normal. The persistence of such inflammation may contribute to high rates of appendicitis recurrence, and chronic inflammation is one of the risk of canceration. Interval appendectomy is considered necessary to reduce the risk of relapse of appendicitis and prevent canceration.

Our study had several limitations. First, this study was not a randomized study but a retrospective study at a single center, and the number of patients was small. Second, surgery and perioperative management were performed by different clinicians. Although the operative procedure may vary depending on the skill of operators, it may be possible for trained surgeons to perform laparoscopic appendectomy in the elective surgery after conservative therapy for appendicitis.

As a future prospect, further accumulation of cases with complicated appendicitis and randomized studies are expected to investigate the usefulness of interval appendectomy. It is also considered necessary to examine the difference in residual inflammation depending on the timing of interval appendectomy.

\section{Conclusions}

In our study of 38 patients who received initial conservative treatment for complicated appendicitis, the interval appendectomy protocol was completed in $89 \%$ of cases except for cases in which cancer was detected. Interval appendectomy showed a low incidence of perioperative complications. At the same time, cost-effectiveness of interval appendectomy compared emergency surgery was also demonstrated. Given these findings and residual inflammation in half of the cases on pathological examination, we suggest that interval appendectomy for complicated appendicitis is appropriate and recommended.

\section{References}

[1] Barnes BA, Behringer GE, Wheelock FC, Wilkins EW. Treatment of appendicitis at the Massachusetts General Hospital (1937-1959). JAMA. 1962; 180: 122-6.

[2] Thomas DR. Conservative management of the appendix mass. Surgery 1973; 73: 677-80.

[3] Bradley EL, III, Isaacs J. Appendiceal Abscess Revisited. Arch Surg. 1978; 113: 130-2.

[4] Blakely ML, Williams R, Dassinger MS, Eubanks JW, 3rd, Fischer P, Huang EY, et al. Early vs interval appendectomy for children with perforated appendicitis. Arch Surg. 2011; 146: 660-5.

[5] St Peter SD, Aguayo P, Fraser JD, Keckler SJ, Sharp SW, Leys $\mathrm{CM}$, et al. Initial laparoscopic appendectomy versus initial nonoperative management and interval appendectomy for perforated appendicitis with abscess: a prospective, randomized trial. J Pediatr Surg. 2010; 45: 236-40.

[6] Armstrong J, Merritt N, Jones S, Scott L, Butter A. Non-operative management of early, acute appendicitis in children: is it safe and effective? J Pediatr Surg. 2014; 49: 782-5.

[7] Tiwari MM, Reynoso JF, Tsang AW, Oleynikov D. Comparison of outcomes of laparoscopic and open appendectomy in management of uncomplicated and complicated appendicitis. Ann Surg 2011; 254: 927-32.

[8] Rice-Townsend S, Hall M, Barnes JN, Baxter JK, Rangel SJ. Hospital readmission after management of appendicitis at freestanding children's hospitals: contemporary trends and financial implications. J Pediatr Surg. 2012; 47: 1170-6.

[9] Cash CL, Frazee RC, Abernathy SW, Childs EW, Davis ML, Hendricks JC, et al. A prospective treatment protocol for outpatient laparoscopic appendectomy for acute appendicitis. J Am Coll Surg. 2012; 215: 101-5; discussion 105-6.

[10] Mosegaard A, Nielsen OS. Interval appendectomy. A retrospective study. Acta Chir Scand. 1979; 145: 109-11.

[11] Paull DL, Bloom GP. Appendiceal abscess. Arch Surg. 1982; 117: 1017-9.

[12] Al-Kurd A, Mizrahi I, Siam B, Kupietzky A, et al. Outcomes of interval appendectomy in comparison with appendectomy for acute appendicitis. The Journal of surgical research 2018; 225: 90-94.

[13] Cheng Y, Xiong X, Lu J, Wu S, Zhou R, Cheng N. Early versus delayed appendicectomy for appendiceal phlegmon or abscess. Cochrane Database Syst Rev 2017; 6: Cd011670.

[14] Talan DA, Saltzman DJ, Mower WR, Krishnadasan A, Jude $\mathrm{CM}$, Amii R, et al. Antibiotics-first versus surgery for appendicitis: A US pilot randomized controlled trial allowing outpatient antibiotic management. Ann Emerg Med 2017; 70: 1-11. e19. 
[15] Perez KS, Allen SR. Complicated appendicitis and considerations for interval appendectomy. journal of the American Academy of Physician Assistants. 2018; 31 (9): 35-41.

[16] Miyo M, Urabe S, Hyuga S, et al. Clinical outcomes of single-site laparoscopic interval appendectomy for severe complicated appendicitis: Comparison to conventional emergency appendectomy. Annals of gastroenterological surgery $2019 ; 3$ (5): 561-567.

[17] Lai HW, Loong CC, Wu CW, Lui WY. Watchful waiting versus interval appendectomy for patients who recovered from acute appendicitis with tumor formation: a cost-effectiveness analysis. J Chin Med Assoc 2005; 68: 431-4.
[18] Luo CC, Cheng KF, Huang CS, Lo HC, Wu SM, Huang HC, et al. Therapeutic effectiveness of percutaneous drainage and factors for performing an interval appendectomy in pediatric appendiceal abscess. BMC Surg. 2016; 16: 72.

[19] Tannoury J, Abboud B. Treatment options of inflammatory appendiceal masses in adults. World J Gastroenterol 2013; 19: 3942-50.

[20] Dina Fouad, Jeremy D. Kauffman, Nicole M. Chandler. Pathology findings following interval appendectomy: Should it stay or go? J Pediat Surg. 2020; 55 (4): 737-741.

[21] Rosen M, Chalupka A, Butler K, et al. Pathologic findings suggest long-term abnormality after conservative management of complex acute appendicitis. Am Surg. 2015; 81 (3): 297-299. 